\title{
BERGE'S MAXIMUM THEOREM WITH TWO \\ TOPOLOGIES ON THE ACTION SET
}

\author{
by \\ Anthony Horsley and A J Wrobel \\ Department of Economics, London School of Economics \\ and \\ Timothy Van Zandt \\ Department of Economics, Princeton University
}

Contents:

Abstract

1. Introduction

2. Two-Topology Maximum Theorems

3. Application to Consumer Demand References

Discussion Paper

No.TE/98/347

March 1998
The Suntory Centre

Suntory and Toyota International Centres

For Economics and Related Disciplines

London School of Economics and Political

Science

Houghton Street

London WC2A 2AE

Tel.: 0171-405 7686 


\section{Abstract}

We give variants on Berge's Maximum Theorem in which the lower and the upper semicontinuities of the preference relation are assumed for two different topologies on the action set, i.e., the set of actions available a priori to the decision-maker (e.g. a household with its consumption set). Two new uses are pointed to. One result, stated here without a detailed proof, is the norm-to-weak continuity of consumer demand as a function of prices (a property pointed to in existing literature but without proof or precise formulation). This improves significantly upon an earlier demand continuity result which, with the extremely strong "finite" topology on the price space, is of limited interest other than as a vehicle for an equilibrium existence proof. With the norm topology on the price space, our demand continuity result acquires an independent significance - particularly for practical implementations of the equilibrium solution. The second application referred to established the continuity of the optimal plan as a function of the decision-maker's information (represented by a field of events in a probability space of states).

Keywords: Berge's Maximum Theorem; demand continuity. JEL Nos.: C61, C62.

(C) by Anthony Horsley, Timothy van Zandt and A J Wrobel. All rights reserved. Short sections of text, not to exceed two paragraphs, may be quoted without explicit permission provided that full credit, including (c) notice, is given to the source. 


\section{INTRODUCTION}

The set of actions available a priori to a decision-maker can carry more than one topology relevant to the problem; and in applying Berge's Maximum Theorem it can be important to relax the continuity assumption on the preference preorder $\preccurlyeq$ for the topology $\mathcal{W}$ that makes the set of actions $X$ compact. This is so in, e.g., equilibrium analysis set up in infinite-dimensional commodity spaces, since even some of the simplest functional forms of utility are $\mathcal{W}$-discontinuous. For example, an additively separable, strictly concave utility function on $L_{+}^{\infty}$ (or a subset $X$ thereof) is not weakly* lower semicontinuous (l.s.c.), although it is Mackey-continuous and hence weakly* upper semicontinuous (u.s.c.): see [3, Appendix II]. The lower semicontinuity of preferences should therefore be assumed for an auxiliary topology $\mathcal{S}$ that is, in general, significantly stronger than the "compactifying" topology $\mathcal{W}$ (although in principle $\mathcal{S}$ might be incomparable to $\mathcal{W}$, being stronger only in some respects).

Such problems require, then, an extension of the Maximum Theorem to the case of a preference preorder which is $\mathcal{W}$-u.s.c. but only $\mathcal{S}$-l.s.c., where $\mathcal{W}$ and $\mathcal{S}$ are two topologies on the set of actions. The topology used on the set of parameters (a.k.a. environments) is denoted by $\mathcal{P}$. Section 2 of this note provides a variety of such extensions. It starts with a basic yet applicable form of the result, resting on minimal assumptions - which do not require the action set $X$ to be compact, or the constraint $B$ to be closed (Theorem 2.2). As assumptions are added, the variant ready-made for a proof of consumer demand continuity, as a function of prices, is arrived at (Corollary 2.8). This application is presented in Section 3. Demand continuity in consumer characteristics can also be established by these methods; and it is for this reason that the basic results are stated for parameter-dependent preferences - as in, e.g., the exposition [9, 9.2.4] of the Maximum Theorem.

Apart from strengthening the result, the use of two different topologies on $X$ helps bring out the structure of the Maximum Theorem and the arguments employed to prove it, which remain essentially the same as Berge's [2, pp. 115-116]. For conclusions similar to those of the original version, the topology $\mathcal{W}$ must be weak enough to make the action set compact and, also, to make the constraint correspondence $\mathcal{P}$-to- $\mathcal{W}$ upper hemicontinuous (u.h.c.), or at least closed. As for $\mathcal{S}$, it enters the assumptions but not the conclusion - which is that the optimal action correspondence (a.k.a. choice or decision correspondence) is $\mathcal{P}$-to- $\mathcal{W}$ u.h.c. The topology $\mathcal{S}$ is thus purely auxiliary: its role is solely to make the constraint correspondence $\mathcal{P}$-to- $\mathcal{S}$ lower hemicontinuous (1.h.c.) whilst making preferences $\mathcal{S}$-l.s.c. It must be strong enough to meet the latter (semi-continuity) condition but not so strong as to fail the former (hemi-continuity) condition. Specification of $\mathcal{S}$ is otherwise immaterial; and in principle $\mathcal{S}$ need not be stronger than $\mathcal{W}$ or even comparable with it. As for $\mathcal{P}$ (the parameter-space topology), this should of course be kept as weak as possible (i.e., just strong enough to make the constraint correspondence both $\mathcal{P}$-to- $\mathcal{W}$ u.h.c. and $\mathcal{P}$-to-S l.h.c.). 


\section{Two-topology Maximum Theorems}

This section gives a progression of variants on Berge's Theorem for the case of a preference relation $R$ that is u.s.c. for one topology, $\mathcal{W}$, and l.s.c. for another, $\mathcal{S}$. This implies that the graph, gr $R$, of $R$ is $(\mathcal{S} \times \mathcal{W})$-closed, if $R$ is a total weak preorder in the set of actions $X$ (Lemma 2.6). But no such assumption is made on $R$ to start with, and so its $(\mathcal{S} \times \mathcal{W})$-closedness is assumed directly at first. Apart from this, the given variants of Berge's Theorem differ from each other in the continuity assumptions on the constraint correspondence $B$-which maps the points of the parameter space $P$ to $\mathcal{W}$-closed subsets of $X$-and in the corresponding conclusions about the optimum-choice correspondence $\hat{X}$. At first, nothing but $\mathcal{P}$-to-S lower hemicontinuity is assumed of $B$; and then the conclusion is that, for a $(\mathcal{P} \times \mathcal{W})$-limit of parameteroptimum pairs, its feasibility is sufficient for optimality-i.e., a $(\mathcal{P} \times \mathcal{W})$-limit point of the graph of $\hat{X}$ is itself a point thereof if only it lies in the graph of $B$ (Theorem 2.2 ). It follows that if, in addition, the constraint graph is $(\mathcal{P} \times \mathcal{W})$-closed, then so is the optimum-choice graph (Corollary 2.3). If the constraint $B$ meets the stronger condition of upper hemicontinuity with compact values, then so does the choice $\hat{X}$ (Corollary 2.4). These results can clearly be combined in a number of useful ways, but only the one needed for the application to continuity of consumer demand in prices (Section 3) is recorded here (Corollary 2.8).

One use of demand continuity is for equilibrium existence, and in that context also the "minimalist" variant (Theorem 2.2) comes in handy. This is because the two weak* topologies put on the price set $P$ and the consumption set $X$ for the fixed-point argument are too weak to make the budget constraint closed: see [6]. ${ }^{1}$

Let $B$ be a correspondence from a topological space $(P, \mathcal{P})$ into a set $X$ carrying two topologies, $\mathcal{S}$ and $\mathcal{W}$. All three are assumed to be Hausdorff topologies (T2), and $\mathcal{W}$ is assumed to be regular (T3). Though this is not always needed, every T1 vector-space topology is actually T3. In the existing applications (Section 3 here and $[11]), \mathcal{S}$ is stronger than $\mathcal{W}$ (whence the notation).

Since the terminology varies in the literature, hemicontinuity of a correspondence is defined next. Semicontinuity - a term we reserve for orderings (and real-valued functions) - is introduced later.

Definition 2.1. A correspondence $C$ from $P$ into $X$ is:

1. $\mathcal{P}$-to-S lower hemicontinuous (l.h.c.) if, for each $\mathcal{S}$-open set $S$, its lower inverse image $\{p: C(p) \cap S \neq \emptyset\}$ is $\mathcal{P}$-open;

2. $\mathcal{P}$-to-S upper hemicontinuous if, for each $\mathcal{W}$-open set $W$, its upper inverse image $\{p: C(p) \subseteq W\}$ is $\mathcal{P}$-open.

\footnotetext{
${ }^{1}$ That paper merges our earlier manuscripts, circulated at Princeton University and the LSE in 1988-89 (and later as STICERD Discussion Paper TE/92/246). It is in those that two-topology extensions of Berge's Theorem first appeared.
} 
For the basic results the preference relation in $X$ is allowed to depend on the parameter $p \in P$, i.e., $R$ is actually a subset of $X \times P \times X$. It defines, for each $p \in P$, a binary relation $R_{p}$ in $X$ by

$$
z R_{p} x \Leftrightarrow(z, p, x) \in R .
$$

The choice correspondence is then defined, for $p \in P$, by

$$
\hat{X}(p):=\left\{x \in B(p): \forall z \in B(p) z R_{p} x\right\} .
$$

The first result, apart from being fundamental for this analysis, is useful when $\mathcal{P} \times \mathcal{W}$ is too weak to make the constraint $B$ closed.

Theorem 2.2. Assume that $B$ is $\mathcal{P}$-to-S lower hemicontinuous, and that $R$ is $(\mathcal{S} \times \mathcal{P} \times \mathcal{W})$-closed (in $X \times P \times X)$. Then, for every $(p, x)$ in the $(\mathcal{P} \times \mathcal{W})$-closure of $\operatorname{gr} \hat{X}$ in $P \times X$, if $x \in B(p)$, then $x \in \hat{X}(p)$.

Proof. If $x \in B(p)$ but $x \notin \hat{X}(p)$, then $(z, p, x) \notin R$ for some $z \in B(p)$. So there exist: a $\mathcal{S}$-neighbourhood, $N_{z}$, of $z$, a $\mathcal{P}$-neighbourhood, $N_{p}$, of $p$ and a $\mathcal{W}$-neighbourhood, $N_{x}$, of $x$ such that $\left(z^{\prime}, p^{\prime}, x^{\prime}\right) \notin R$ for every $z^{\prime} \in N_{z}, p^{\prime} \in N_{p}$ and $x^{\prime} \in N_{x}$. Since $B$ is $\mathcal{P}$-to-S l.h.c., there also exists a $\mathcal{P}$-neighbourhood, $H_{p}$, of $p$ such that $B\left(p^{\prime}\right) \cap N_{z} \neq \varnothing$ for every $p^{\prime} \in H_{p}$. It follows that $x^{\prime} \notin \hat{X}\left(p^{\prime}\right)$ for every $x^{\prime} \in N_{x}$ and every $p^{\prime} \in H_{p} \cap N_{p}$; and this shows that $(p, x)$ does not belong to the $(\mathcal{P} \times \mathcal{W})$-closure of $\operatorname{gr} \hat{X}$.

It follows that closedness (of the graph) is a condition that, when assumed of $B$, "reproduces" itself as a property of $\hat{X}$.

Corollary 2.3. On the assumptions of Theorem 2.2, if $\operatorname{gr} B$ is $(\mathcal{P} \times \mathcal{W})$-closed (in $P \times X)$, then so is $\operatorname{gr} \hat{X}$.

Proof. Take any $(p, x)$ in the $(\mathcal{P} \times \mathcal{W})$-closure of gr $\hat{X}$. A fortiori, $(p, x)$ belongs to the $(\mathcal{P} \times \mathcal{W})$-closure of gr $B$, which equals gr $B$ by assumption; so $x \in B(p)$. Therefore $x \in \hat{X}(p)$ by Theorem 2.2 .

Like closedness, the stronger property of upper hemicontinuity with compact values turns out to "propagate" from $B$ to $\hat{X}$. (This is a stronger property because a u.h.c. correspondence with closed values has a closed graph: see, e.g., [9, 7.1.15].)

Corollary 2.4. On the assumptions of Corollary 2.3, if $B$ is $\mathcal{P}$-to- $\mathcal{W}$ upper hemicontinuous with $\mathcal{W}$-compact values, then so is $\hat{X}$.

Proof. The correspondence obtained as the intersection of one with a closed graph and another that is u.h.c. with compact values is also u.h.c.: see, e.g., [9, 7.3 .10 (ii)]. Here this is applied to $\hat{X}=B \cap \hat{X}$, with $\hat{X}$ being closed by Corollary 2.3.

For the rest of this section, $\preccurlyeq$ denotes a weak preorder (i.e., a reflexive and transitive relation) in $X$. The associated strict preorder in $X$ is defined by

$$
z \succ x \Leftrightarrow(z \succcurlyeq x \text { and } z \npreceq x) .
$$


This is an irreflexive and transitive relation in $X$.

In the context of consumer demand, a parameter point $p$ may specify not only the market price system $\pi$ but also consumer characteristics - indeed, the consumer preference relation $\preccurlyeq$ itself can be viewed as a parameter. In that case $p$ is formally the pair $(\pi, \preccurlyeq)$, and the budget set $B(\pi)$ depends on $\pi$ but not on $\preccurlyeq$, whilst $R=\{(z, \preccurlyeq, x): z \preccurlyeq x\}$, i.e., $R_{\pi, \preccurlyeq}$ is simply $\preccurlyeq$. Since $R$ can depend on $p$ in the preceding results, these can be used to establish the continuity of demand in consumer characteristics $(\preccurlyeq)$ as well as in prices $(\pi)$. However, since only the price-continuity result is stated here, for the rest of this section $\preccurlyeq$ is a fixed (parameter-independent) preorder in the action set $X$; and so $\hat{X}$ is henceforth defined by 2.1 with $\nsucc$ in place of $R_{p}$. Note, also, that $\nsucc$ equals $\preccurlyeq$ if and only if $\preccurlyeq$ is total (a.k.a. complete, i.e., $x^{\prime} \preccurlyeq x^{\prime \prime}$ or $x^{\prime \prime} \preccurlyeq x^{\prime}$ for each $x^{\prime}$ and $x^{\prime \prime}$ in $X$ ).

Definition 2.5. An irreflexive and transitive relation $\succ$ in $X$ is:

1. S-lower semicontinuous if, for each $z \in X$, the set $\{x \in X: x \succ z\}$ is $\mathcal{S}$-open (in $X$ ).

2. $\mathcal{W}$-upper semicontinuous if the set $\{x \in X: z \succ x\}$ is $\mathcal{W}$-open, for each $z \in X$.

Comment: For a total $\preccurlyeq$, lower semicontinuity of $\succ$ is equivalent to closedness of the set $\{x: x \preccurlyeq z\}$ for each $z$, and is also referred to as l.s.c. of $\preccurlyeq$. Similarly, upper semicontinuity of $\succ$ is equivalent to closedness of the set $\{x: z \preccurlyeq x\}$ for each $x$, and is referred to as u.s.c. of $\preccurlyeq$.

Some well-known conditions under which openness of the graph (of $\succ$ ) follows from that of its sections (i.e., from the two semicontinuities) are spelt out next.

Lemma 2.6. Assume that $\succ$ is $\mathcal{S}$-lower semicontinuous and $\mathcal{W}$-upper semicontinuous. Then:

1. If $\succ$ is also dense (i.e., for every $x$ and $z$ in $X$ with $z \succ x$ there exists $a y \in X$ with $z \succ y \succ x)$, then the graph of $\succ$ is $(\mathcal{S} \times \mathcal{W})$-open.

2. $\succ$ is dense if $\preccurlyeq$ is total and $X$ is connected for the topology $\mathcal{S} \vee \mathcal{W}$.

Proof. For Part 1 , if $z \succ x$, then $z \succ y \succ x$ for some $y$. So there exists a $\mathcal{S}$ neighbourhood, $N_{z}$, of $z$ and a $\mathcal{W}$-neighbourhood, $N_{x}$, of $x$ such that for every $z^{\prime} \in N_{z}$ and $x^{\prime} \in N_{x}$ one has $z^{\prime} \succ y \succ x^{\prime}$ and therefore $z^{\prime} \succ x^{\prime}$ by transitivity.

For Part 2, if $z \succ x$, then consider the $\mathcal{S}$-open set $\{y: y \succ x\}$ and the $\mathcal{W}$-open set $\{y: z \succ y\}$. If they were not disjoint, then their union could not equal $X$ (since $X$ is connected); i.e., there would exist a $y \in X$ with $z \nsucc y$ and $y \nsucc x$. Since $\preccurlyeq$ is total, this is equivalent to $z \preccurlyeq y \preccurlyeq x$, which implies $z \preccurlyeq x$ by transitivity (and thus contradicts $z \succ x$ ).

The following version of Weierstrass' Theorem is contained in, e.g., the formulation [9, 9.2.4] of the Maximum Theorem.

Proposition 2.7. If $\succ$ is $\mathcal{W}$-upper semicontinuous, and the values of $B$ are $\mathcal{W}$ compact and nonempty, then so are the values of $\hat{X}$. 
Proof. Suppose that $\hat{X}=\emptyset$ at some $p$, i.e., for each $x \in B$ the set $S(x):=$ $\{z \in B: z \succ x\}$ is nonempty (where, with $p$ fixed, $\hat{X}(p)$ and $B(p)$ are abbreviated to $\hat{X}$ and $B$ ). Then $\{S(x)\}_{x \in B}$ is an open cover of the compact $B$, and so it has a finite subcover; i.e., there is a nonempty finite set $F \subset B$ such that $\forall x \in B \exists z \in F z \succ x$. Starting from any $z_{1} \in F$, choose a sequence $\left(z_{n}\right)$ in $F$ such that $z_{n+1} \succ z_{n}$ for each $n \in \mathbb{N}$. Since $F$ is finite, $x_{m}=x_{n}$ for some $m$ and $n$ with $m>n$. But $x_{m} \succ x_{n}$ by transitivity, so $x_{m} \neq x_{n}$ by irreflexivity; a contradiction.

A special case of interest is that of a $\mathcal{W}$-compact convex subset $X$ of a vector space $L$ with vector topologies $\mathcal{S}$ and $\mathcal{W}$. In this case, since the range is compact, closedness and upper hemicontinuity are equivalent (for a closed-valued correspondence): see, e.g., $[9,7.1 .16]$.

Corollary 2.8. Let $B$ be a correspondence from a topological space $(P, \mathcal{P})$ into a convex subset $X$ of a vector space $L$ with topologies, $\mathcal{S}$ and $\mathcal{W}$ whose restrictions to any straight line in $L$ are both identical to the usual topology of $\mathbb{R}$ (as is the case with vector topologies). Assume that $X$ is $\mathcal{W}$-compact, that the values of $B$ are nonempty and $\mathcal{W}$-closed (and hence compact), and that $B$ is $\mathcal{P}$-to- $\mathcal{W}$ upper hemicontinuous and $\mathcal{P}$-to-S lower hemicontinuous. If, in addition, $\preccurlyeq$ is a total weak preorder in $X$ that is $\mathcal{S}$-lower semicontinuous and $\mathcal{W}$-upper semicontinuous, then the optimal action correspondence, $\hat{X}: P \rightarrow X$, defined by (2.1) with $\preccurlyeq$ in place of $R$, is $\mathcal{P}$-to- $\mathcal{W}$ upper hemicontinuous (with nonempty and $\mathcal{W}$-compact values).

Proof. Apply Lemma 2.6, Corollary 2.3 or 2.4, and Proposition 2.7.

\section{Application to CONSUmer Demand}

This section gives a precise statement of the norm-to-weak* continuity of demand as a function of the price system when the commodity space, $L$, is the norm-dual of a Banach space $L^{\prime}$. More precisely, what can be established is that the demand is a norm-to-weak* upper hemicontinuous correspondence from the price cone (i.e., the polar of the production cone) in $L^{\prime}$ into $L .^{2}$

With $L^{\prime}$ serving as the price space in the demand continuity result, $\langle p, x\rangle$ denotes the value of a commodity bundle $x \in L$ at a price system $p \in L^{\prime}$. This, the normpredual of $L$, is contained in the norm-dual $L^{*}$ of $L$ (which is a larger price space). The norm of a $p \in L^{\prime}$ is denoted by $\|p\|^{\prime}$; the dual norm of an $x \in L$ is $\|x\|$. The weak* topology of $L$ is denoted by w* for brevity; the full notation is w $\left(L, L^{\prime}\right)$. Also, the so-called "finite" topology on the commodity space $L$-in which a set is closed if and only its intersection with any affine subspace of a finite dimension $d$ is closed for

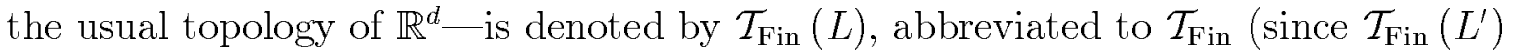
is not used here).

The total production set is taken to be a cone $Y \subset L$. The household's preferences, taken to be complete and transitive, are described by a total weak preorder $\preccurlyeq$ on

\footnotetext{
${ }^{2}$ This result is pointed to (but not stated precisely) in [7, pp. 191 and 193].
} 
the consumption set, $X$. The household's initial endowment is denoted by $x^{\mathrm{En}}$. The household's budget set is therefore

$$
B(p):=\left\{x \in X:\langle p, x\rangle \leq\left\langle p, x^{\mathrm{En}}\right\rangle\right\} .
$$

and its demand is

$$
\hat{X}(p):=\left\{x \in B(p): \forall x^{\prime} \in B(p) x^{\prime} \preccurlyeq x\right\} .
$$

Recall also that the $L^{\prime}$-polar cone of $Y$ is

$$
Y^{\circ} \cap L^{\prime}:=\left\{p \in L^{\prime}: \forall y \in Y\langle p, y\rangle \leq 0\right\},
$$

where $Y^{\circ}$ denotes the algebraic polar. For brevity, denote

$$
\begin{aligned}
P^{*} & :=\left(Y^{\circ} \cap L^{*}\right) \backslash\{0\} \\
P^{\prime} & :=P^{*} \cap L^{\prime}=\left(Y^{\circ} \cap L^{\prime}\right) \backslash\{0\} .
\end{aligned}
$$

Theorem 3.1. Assume that the consumption set $X$ is $\mathrm{w}^{*}$-compact and convex (and nonempty), and that

$$
\forall p \in P^{\prime} \exists x \in X\left\langle p, x-x^{\mathrm{En}}\right\rangle<0,
$$

i.e., that at every technologically possible price system there is an adequate consumption bundle worth less than the endowment. If, furthermore, the preference preorder $\preccurlyeq$ is $\mathrm{w}^{*}$-upper semicontinuous and $\mathcal{T}_{\mathrm{Fin}}$-lower semicontinuous (i.e., for every $x^{\prime}$ the set $\left\{x \in X: x^{\prime} \preccurlyeq x\right\}$ is $\mathrm{w}^{*}$-closed, whilst the set $\left\{x \in X: x \preccurlyeq x^{\prime}\right\}$ is $\mathcal{T}_{\text {Fin }}(L)$-closed), then the consumer demand, $p \mapsto \hat{X}(p)$, is a norm-to-weak ${ }^{*}\left(\|\cdot\|^{\prime}\right.$-to- $\left.\mathrm{w}^{*}\right)$ upper hemicontinuous correspondence from $P^{\prime}$ into $X$ (with $\mathrm{w}^{*}$-compact and nonempty values).

Proof. This can be proved by an application of Corollary 2.8 , with $\mathcal{W}=\mathrm{w}^{*}, \mathcal{S}=\mathcal{T}_{\text {Fin }}$, and $\mathcal{P}$ defined by $\|\cdot\|^{\prime}$ on $P^{\prime}$ : see $[6]$ for details.

\section{Comments:}

1. One use of demand continuity is in equilibrium existence proofs, but its significance goes beyond this. For example, in practical implementations of the equilibrium solution it is essential to know that small deviations from the equilibrium price system - caused either by errors or by deliberate simplification of a complex exact solution - will not result in large shifts of demand. However, if demand continuity is to be of such an additional interest, the topology $\mathcal{P}$ of the price space must be kept as weak as possible. If, by contrast, an extremely strong topology is used on the price space, then demand continuity becomes a rather weak result which cannot be of much interest other than as a vehicle for proving equilibrium existence. This is the case with [4], where the price space carries the finite topology $\mathcal{T}_{\text {Fin }}\left(L^{\prime}\right)$, which is even stronger than $\mathcal{T}_{\mathrm{SV}}\left(L^{\prime}\right)$, the strongest vector topology. It is our choice of the norm topology for $\mathcal{P}$ in applying the (extended) Maximum Theorem that allows us to improve on the continuity result of [4, Proof of Proposition 3]. A significantly weaker choice of $\mathcal{P}$ would not 
do: demand can be weak-to-weak* (i.e., w $\left(L^{\prime}, L\right)$-to-w* $\left.{ }^{*}\right)$ discontinuous, as an example in $[6]$ shows.

2. The finite topology on the commodity space is the best choice for $\mathcal{S}$ in the context of consumer demand. It gives a very large class of continuous preferences, which obviously includes all the norm-continuous ones. And actually the $\mathcal{T}_{\text {Fin }}{ }^{-}$ continuity condition is no more restrictive than it is in the finite-dimensional case (so the only truly "infinite-dimensional" restriction on preferences is that of $\mathrm{w}^{*}$-u.s.c. here). This very strong choice of $\mathcal{S}$ works because the budget correspondence is norm-to- $\mathcal{T}_{\text {Fin }}$ l.h.c.: see [6]. However, the distinction between $\mathcal{T}_{\text {Fin }}$ and the norm topology (of $L$ ) is rather insignificant in applications; and little would be lost by assuming the l.s.c. of preferences for the norm (instead of $\left.\mathcal{T}_{\text {Fin }}\right)$.

3. Note, however, that $\mathcal{T}_{\text {Fin }}$ is not a vector topology, unless $\operatorname{dim} L$ is countable (which is never the case for an infinite-dimensional Banach space $L$ ): see, e.g., [8, Section 3: p. 108]. When the vector-space property, or local convexity, is also needed, the best choice for $\mathcal{S}$ is the strongest vector topology $\mathcal{T}_{\mathrm{SV}}$, or the strongest locally convex topology $\mathcal{T}_{\text {SLC }}$. Even with $\mathcal{T}_{\text {SLC }}$ on $L$, every concave function $U: L \rightarrow \mathbb{R}$ is continuous: see, e.g., [1, V.3.3 (d)].

4. The weak* compactness condition on the consumption set can be met by means of truncation, as is done in equilibrium existence proofs (under the boundedness assumption on the set of feasible allocations). But it can be justified quite generally - also out of equilibrium - by physical constraints on consumption or simply by eventual satiation (since the nonsatiation property, when required, need be assumed only at the attainable bundles).

5. The Adequacy Assumption (3.2) of Theorem 3.1 obviously holds if

$$
\left(X-x^{\mathrm{En}}\right) \cap \operatorname{core}(Y) \neq \varnothing,
$$

i.e., if a feasible trade for the consumer belongs to the production cone's core. In a Banach space $L$, the core of a convex, norm-closed set $Y$ is equal to the norm-interior of $Y$ : see, e.g., [5, p. 84] or [10, II.7.1]. For a cone $Y$ in $L$ with a nonempty core it follows that $Y^{\circ} \subset L^{*}$, and so (3.1) simplifies to $P^{*}:=Y^{\circ} \backslash\{0\}$.

6. Condition (3.3) also guarantees that $P^{*}$ has a w $\left(L^{*}, L\right)$-compact base, which can be used for a fixed-point argument in proving the existence of an equilibrium (with a price system $p^{\star} \in L^{*}$ ). The existence result requires, of course, additional assumptions (viz., the convexity of preferences and of the production set $Y$, and also the $\mathrm{w}^{*}$-closedness of $Y$ ). 


\section{REFERENCES}

[1] Bair, J., and R. Fourneau (1975): Etude geometrique des espaces vectoriels (Lecture Notes in Mathematics, vol. 489). New York-Heidelberg-Berlin: Springer-Verlag.

[2] Berge, C. (1963): Topological spaces. Edinburgh: Oliver and Boyd.

[3] Bewley, T. (1972): "Existence of equilibria in economies with infinitely many commodities", Journal of Economic Theory, 4, 514-540.

[4] Florenzano, M. (1983): "On the existence of equilibria in economies with an infinite dimensional commodity space", Journal of Mathematical Economics, 11, 207-219.

[5] Holmes, R. B. (1975): Geometric functional analysis and its applications. New York-HeidelbergBerlin: Springer-Verlag.

[6] Horsley, A., T. Van Zandt, and A. J. Wrobel (1998): "Continuity of demand and the direct approach to equilibrium existence for Banach commodity spaces", extension of STICERD Discussion Paper TE/92/246, LSE.

[7] Jones, L. E. (1986): "Special problems arising in the study of economies with infinitely many commodities", in Models of economic dynamics (Lecture Notes in Economics and Mathematical Systems, vol. 264, pp. 184-205), ed. by H. Sonnenschein. Berlin-Heidelberg-New York-Tokyo: Springer-Verlag.

[8] Klee, Jr., V. L. (1953): "Convex sets in linear spaces. III", Duke Mathematical Journal, 20, $105-111$.

[9] Klein, E., and A. C. Thompson (1984): Theory of correspondences. New York-ChichesterBrisbane-Toronto-Singapore: Wiley.

[10] Schaefer, H. H. (1971): Topological vector spaces. New York-Heidelberg-Berlin: SpringerVerlag.

[11] Van Zandt, T. (1989): "Information, measurability and continuous behaviour", manuscript, Princeton University.

(Anthony Horsley and Andrew J. Wrobel) Department of Economics, London School of Economics, Houghton Street, London WC2A 2AE, United Kingdom

E-mail address: LSEecon1230aol.com

(Timothy Van Zandt) Department of Economics, Princeton University, Princeton, NJ 08544-1021, United States

E-mail address: tvz@princeton. edu 